The Astrophysical Journal, 187 :L63-L66, 1974 January 15

(c) 1974. The American Astronomical Society. All rights reserved. Printed in U.S.A.

\title{
MOLECULAR CLOUDS IN THE GALACTIC NUCLEUS
}

\author{
N. Z. SCOVILLE* AND P. M. Solomon $\dagger$ \\ University of Minnesota \\ AND \\ K. B. JEFFERTS \\ Bell Telephone Laboratories \\ Received 1973 October 9; revised 1973 November 12
}

\section{ABSTRACT}

Observations of the 2.6- $\mathrm{mm}$ CO line have been obtained along the galactic equator covering longitudes $|l| \leq 3^{\circ}$ and velocities $|V| \leq 300 \mathrm{~km} \mathrm{~s}^{-1}$. The strongest emission is concentrated toward the center $(|l|<1.5)$ and at low velocities $\left(<125 \mathrm{~km} \mathrm{~s}^{-1}\right)$. The similarities in distribution and brightness temperature between the $\mathrm{CO}$ and $100-\mu$ emissions suggest that the grains and the gas are colocated and nearly in thermal equilibrium. Kinematic models for the gas in the nucleus are briefly discussed. The mass of this gas (mostly $\mathrm{H}_{2}$ ) is estimated as $10^{7}-10^{8} M_{\odot}$ within $600 \mathrm{pc}$ of the center. This large mass of interstellar molecules indicates that the galactic nucleus may not be deficient in Population I material.

Subject headings: galactic nuclei - galactic structure - molecules, interstellar - radio radiation

\section{INTRODUCTION AND OBSERVATION}

The extraordinarily large abundance of molecules in the galactic-center region has been well established by surveys of $18-\mathrm{cm} \mathrm{OH}$ and 6- $\mathrm{cm} \mathrm{H}_{2} \mathrm{CO}$ absorption (McGee 1970 and Scoville, Solomon, and Thaddeus 1972). Within just several relatively small molecular clouds alone the inferred mass exceeds 10 percent of the 21-cm estimates $\left(4 \times 10^{6} M_{\odot}\right.$, Rougoor 1964) for the entire nucleus $(\omega<750 \mathrm{pc})$. Although most of these molecular clouds are near the center $(\omega \simeq 250 \mathrm{pc})$, their kinematics bear little resemblance to the rotating disk which so successfully accounts for the $\mathrm{H}$ I velocity distribution at $\omega>250 \mathrm{pc}$. Their dominant motion is radial, probably outflow, rather than circular (Scoville 1972).

We report here the first results from an extensive CO line survey of the nucleus covering $|l| \leq 3^{\circ}$, $|V| \leq 300 \mathrm{~km} \mathrm{~s}^{-1}$, and $|b| \leq 0.5$. (The observed grid is crude; the angular spacing is $10^{\prime}$ although the telescope beam is $2^{\prime}$ and integrations were short, resulting in a high noise level, $\sim 0.25^{\circ} \mathrm{K}, T_{A}$.) Unlike the previous $\mathrm{H}_{2} \mathrm{CO}$ and $\mathrm{OH}$ observations of absorption lines there is no bias towards continuum sources in mapping $\mathrm{CO}$ emission at $2.6 \mathrm{~mm}$, and a true picture of the distribution of molecular clouds can be obtained.

The data were obtained in early 1973 with the 16foot $(5-\mathrm{m})$ antenna (HPBW $\left.=2^{\prime}\right)$ of the MillimeterWave Observatory, ${ }^{1}$ a front-end receiver developed at

* Present address: Owens Valley Radio Observatory, California Institute of Technology, Pasadena, California 91109.

$\dagger$ Present address: The Institute for Advanced Study, Princeton, New Jersey 08540.

1 The Millimeter-Wave Observatory is operated by the Electrical Engineering Research Laboratory, The University of Texas at Austin, with support from the National Aeronautics and Space Administration, The National Science Foundation, and McDonald Observatory.
Bell Telephone Laboratories, and a 40-channel filter bank $\left(5.2 \mathrm{~km} \mathrm{~s}^{-1}\right.$ per channel). Correcting the observed antenna temperatures $T_{A}$, for atmospheric attenuation, we find a $T_{A}{ }^{\prime} \sim 2 T_{A}$ at typical elevations of the galactic center (see Davis and Vanden Bout 1973). The equivalent Planck brightness temperature $T_{B}$ can be calculated from $T_{A}{ }^{\prime}$ using equation (5a) of Scoville and Solomon (1973). The desired velocity coverage necessitated two or three observations at each position or 93 observations at each latitude. The synthesized spectra for the completed strip at $b=0^{\circ}$ make up a single longitude-velocity contour diagram (fig. 1).

\section{DISCUSSION}

The bulk of the $\mathrm{CO}$ emission in figure 1 is strongly concentrated within $|V|<125 \mathrm{~km} \mathrm{~s}^{-1}$ and $|l|<1.5$. The emission is also slightly nonaxisymmetric in the sense that there is about 25 percent more integrated intensity at positive longitudes than negative longitudes. The most intense features are at $l=0.17$, $V=60 \mathrm{~km} \mathrm{~s}^{-1} ; l=0.67, V=90 \mathrm{~km} \mathrm{~s}^{-1}$; and $l=$ $-0.67, V=-110 \mathrm{~km} \mathrm{~s}^{-1}$. The first two are extensions of the "Sgr A" and Sgr B2 clouds and will not be discussed further since they are subjects of separate, more detailed, CO studies (Solomon et al. 1972 and Scoville, Solomon, and Penzias 1974). The last, barely detected in molecular absorption, stands out remarkably in CO. It is perhaps identifiable with the farinfrared source Sgr IRC.

The enormous contrast in the relative abundance $\left[\mathrm{H}_{2}\right] /[\mathrm{H} \mathrm{I}]$ between normal spiral arms and the galactic center is suggested by a comparison of the zero-velocity emission with that at higher radial velocities. Although the zero-velocity ridge arising from gas in spiral arms accounts for about 90 percent of $21-\mathrm{cm}$ emission (Kerr and Vallak 1967) it is hardly visible in CO. The mo- 


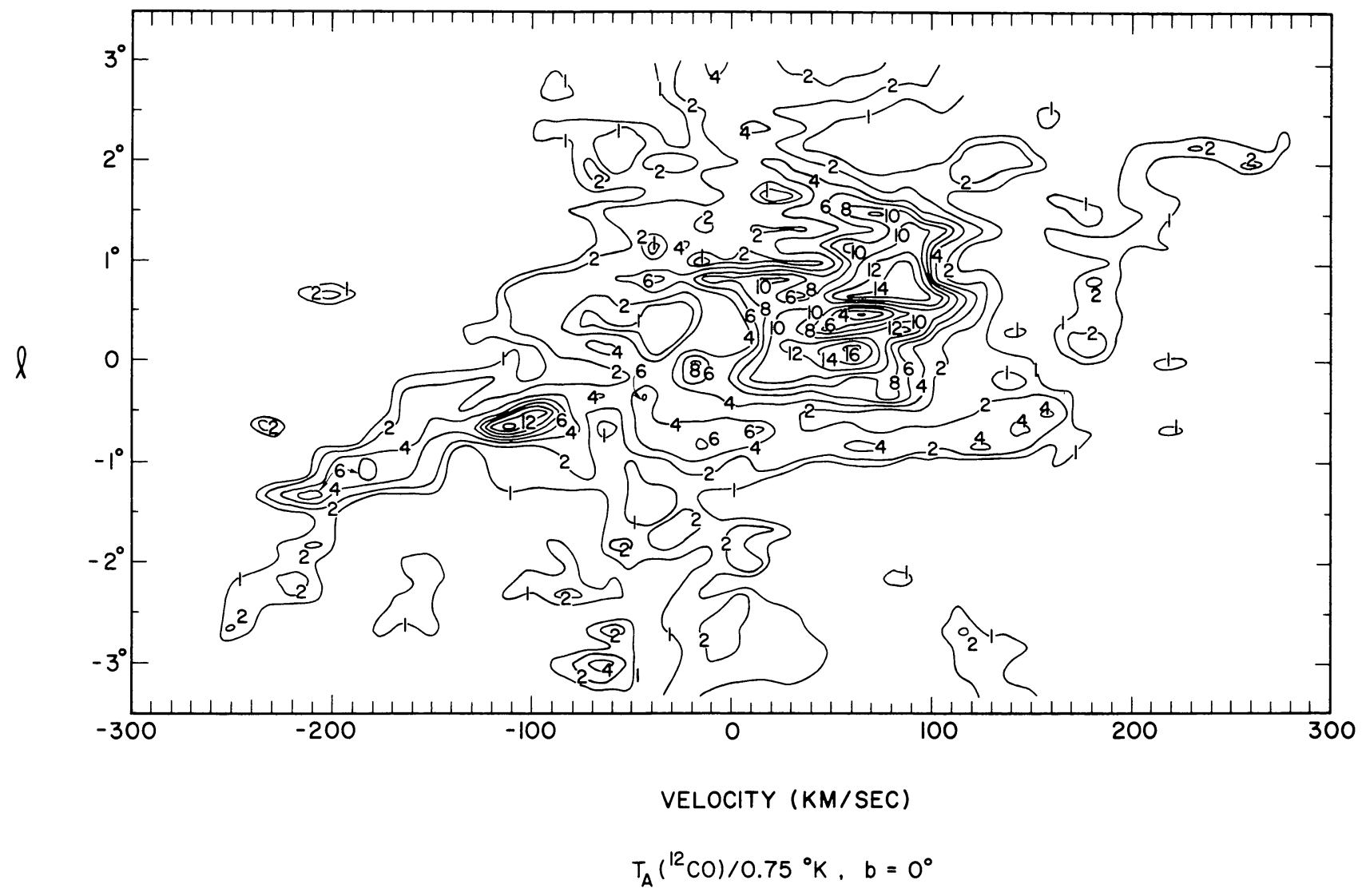

FIg. 1.-Contour diagram of 2.6-mm CO antenna temperature $\left(T_{A}\right)$ in the longitude-velocity plane at $b=0^{\circ}$. Contour unit is $0.75^{\circ} \mathrm{K}$.

lecular abundance in the 3-kpc arm (fig. 1 at $l=-3^{\circ}$ and $-60 \mathrm{~km} \mathrm{~s}^{-1}$ ) is also probably lower than in the nucleus.

\section{a) Nuclear Disk}

The faint $\mathrm{CO}$ emission at $\sim \pm 200 \mathrm{~km} \mathrm{~s}^{-1}$ represents the first detection of distributed molecules corresponding to the 21-cm nuclear-disk feature. Rougoor (1964) encountered the serious problems of inadequate angular resolution and confusion at low velocities, for $|l|<$ $1^{\circ}$. None of the positions observed so far shows strong $\mathrm{CO}$ emission from the high-velocity, outer nuclear disk. The strongest is only $5^{\circ} \mathrm{K}$, at $l=1.2$, and most are much weaker $\left(\sim 1^{\circ} \mathrm{K}\right.$ at $\left.|l|>2^{\circ}\right)$. These lines may be weak because we have chosen a nonoptimum latitude; however, the observations at $b=-20^{\prime},-10^{\prime}$, and $+10^{\prime}$ did not reveal stronger emission.

It is noteworthy that the high-velocity emission $\left(+180 \mathrm{~km} \mathrm{~s}^{-1}\right)$ carries all the way into $l=0.17$. Although we can not rule out the possibility that the emission at $l=-0.5$ and $+150 \mathrm{~km} \mathrm{~s}^{-1}$ arises from the $+135 \mathrm{~km} \mathrm{~s}^{-1} \mathrm{H}$ I arm, it is more likely an extension of the disk feature since a bridge across the $l=0^{\circ}$ gap, continuing on to low velocities at $l=-1^{\circ}$, appears quite certain in the $b=-10^{\prime}$ observations. A nuclear disk which is only rotating clearly cannot account for such behavior.

\section{b) Kinematic Models}

Figure $2 a$ shows schematically and observationally the appearance of the nuclear region as suggested by the previous studies. All structures except the $135 \mathrm{~km}$ $\mathrm{s}^{-1}$ arm were drawn cylindrically symmetric and the nuclear disk was taken as an annulus with inner radius at the ring of molecular clouds. Besides the observational points discussed above, there are also theoretical objections to this multicomponent model (Oort 1970). First, how is it that the nuclear disk remains purely rotating (period $\simeq 10^{7}$ years) when radially moving gas streams only $10^{7}$ years apart and probably containing more mass are found to both the interior and exterior Second the expanding structures require nearly $10^{55}$ ergs for their propulsion and there is not much supporting evidence for such energetic and frequently occurring events in the nucleus.

Bearing these objections in mind and a need for a more unified model we suggest as a possible alternative a two-arm spiral of constant inclination $\left(=23^{\circ}\right)$ with rotation and expansion velocities varying simply with $\omega($ fig. $2 b$ ). Inside of $1 \mathrm{kpc}$ the rotation is rigid body and outside it is with constant velocity. The expansion velocity increases linearly with $\omega$ to $200 \mathrm{~km} \mathrm{~s}^{-1}$ at 750 $\mathrm{pc}$, then falls off. This speculative model accounts for a large body of observations (continuous features and 

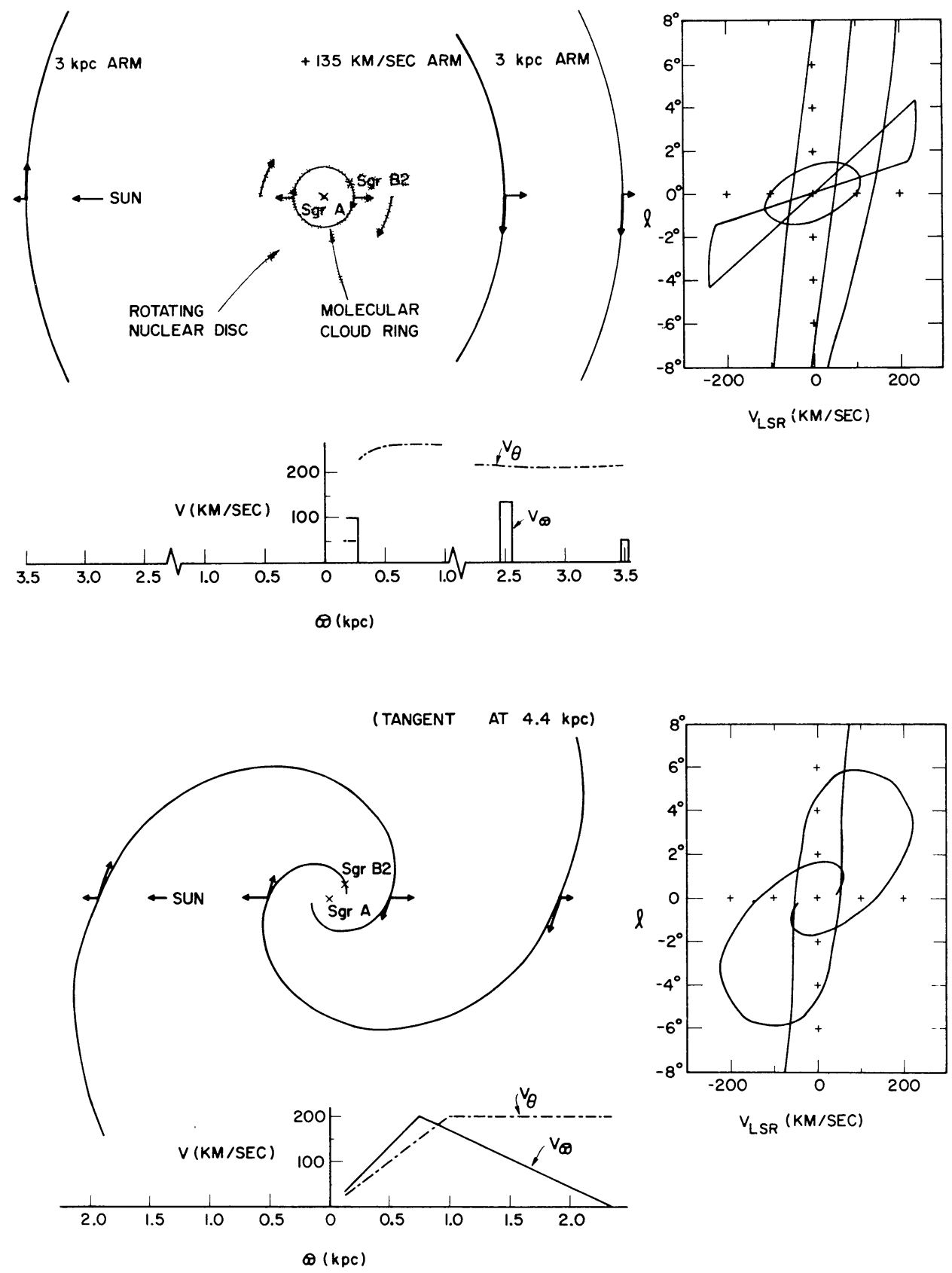

Fig. $2 a(t o p)$.- Sketch (left) of gas structures near the galactic center as seen from above galactic plane and their observational appearance in the longitude-velocity plane (right). Their spatial and kinematic behavior is from Rougoor and Oort (1960), Rougoor (1964), Scoville (1972), and Sanders and Wrixon (1973).

Fig. $2 b$ (bottom).-Sketch and longitude-velocity appearance of the spiral pattern discussed in text as a possible alternative to the model in fig. $2 a$.

their placement in the line of sight relative to continuum sources and each other). Differences in the continuity of features between the two models occur at $l=0^{\circ}$, $V= \pm 135 \mathrm{~km} \mathrm{~s}^{-1}, l= \pm 5^{\circ}, V= \pm 150 \mathrm{~km} \mathrm{~s}^{-1}$, and $l>5^{\circ}, V=200 \mathrm{~km} \mathrm{~s}^{-1}$. Present observational data do not point decisively for or against either model.

\section{c) Comparison with Far-Infrared Observations}

If we regard the $100-\mu$ radiation observed by Hoffmann, Frederick, and Emery (1971) in the galactic center as emission by dust grains, its distribution should closely resemble that of $\mathrm{CO}$ molecules presumably located in dust clouds. To test this hypothesis, the 
100- $\mu$ flux and maximum CO brightness temperature at each longitude are shown in figure 3. Despite the low resolution at $100 \mu\left(=10^{\prime}\right)$ and the coarse spacing of the $\mathrm{CO}$ observations, their spatial variations correlate well; more surprising, the $\mathrm{CO}$ and $100-\mu$ brightness temperatures ( $\sim 20^{\circ} \mathrm{K}$, rightmost scale in fig. 3$)$ are similar at each longitude. Indeed, given the resolution differences and possible calibration errors, the brightness temperatures could be identical. However, the optical depth of the $\mathrm{CO}$ is undoubtedly much greater than the $100-\mu$ grain optical depth. Since the CO brightness temperature is close to the gas kinetic temperature, it appears that the grains are slightly hotter than the gas $\left(T_{\mathrm{gr}} \simeq 30^{\circ}-40^{\circ} \mathrm{K}\right)$ with near thermal equilibrium between grains and gas. The grains which are in thermal equilibrium with the radiation field are then responsible for heating the gas.

\section{d) Mass Distribution}

Although we have not made observations of ${ }^{13} \mathrm{CO}$ emission and therefore cannot directly estimate the $\mathrm{CO}$ optical depth it is possible to derive worthwhile lower limits to the gas density solely on the basis of the $\mathrm{CO}$

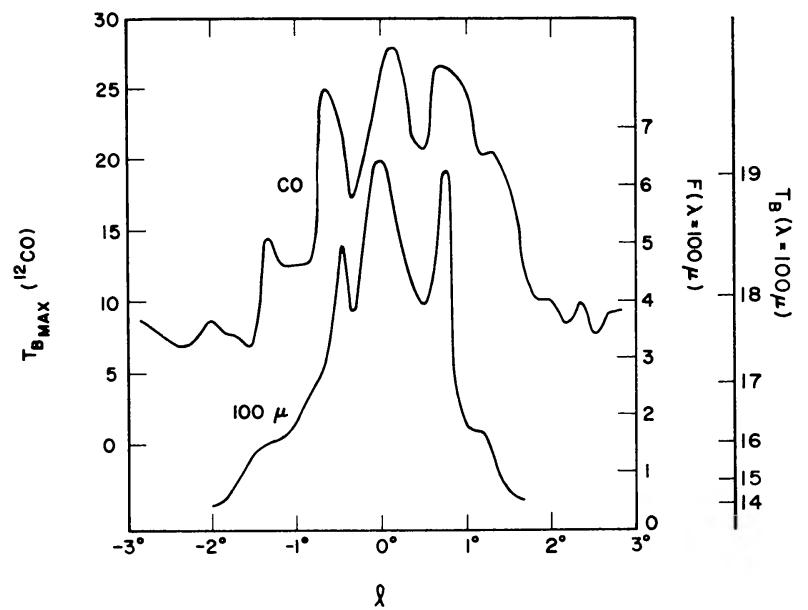

Fig. 3.-The peak brightness temperatures $\left({ }^{\circ} \mathrm{K}\right)$ of the $\mathrm{CO}$ and far-infrared emissions as a function of longitude (at $b=0^{\circ}$ ). The $100-\mu$ flux (Hoffmann et al. 1971) is indicated by the second scale on the right in units of $10^{-3}$ ergs s$\mu^{-1} \mu^{-1} \mathrm{sr}^{-1}$. observations. Providing all CO rotational levels are in thermal equilibrium, the minimum $\mathrm{CO}$ column density consistent with an observed line occurs when the optical depth is $\sim 1 / 2$ (see eq..[7] of Scoville and Solomon 1973). A minimum hydrogen column density consistent with this $\mathrm{CO}$ density is obtained if all the carbon (at normal cosmic abundance) is bound up in $\mathrm{CO}$ and is $3.3 \times 10^{3}$ times the $\mathrm{CO}$ column density.

Integrating in $v$ and $l$ over the emission shown in figure 1 and taking $\Delta b=10^{\prime}$, we find an absolute minimum of $2 \times 10^{6} M_{\odot}$ for the total mass of gaseous hydrogen with about two-thirds of this inside $l=1^{\circ}$. Although this estimate is similar to $\mathrm{H}$ I mass estimates for the nuclear disk $\left(4 \times 10^{6} M_{\odot}\right)$, we cannot overemphasize the degree to which our estimate is conservative. Substantial increases in this estimate occur if the $\mathrm{CO}$ is optically thick and not all the carbon is in $\mathrm{CO}$, both of which are undoubtedly the case. If $\mathrm{CO}$ optical depths are 10 (a typical value derived for regions where the ${ }^{13} \mathrm{CO}$ line has also been observed) instead of 0.5 , the mass increases by a factor of 7 . Taking account of these two factors and a slight increase in the thickness $(\Delta b)$ we feel it realistic to multiply the above number by a factor of 20-50, giving a total mass of $4-10 \times 10^{7} M_{\odot}$ for $\mathrm{H}_{2}$ gas in the nucleus. A similar estimate is obtained on a theoretical basis which requires about $300-1000 \mathrm{H}_{2}$ molecules per $\mathrm{cm}^{3}$ to produce a line of $\sim 3^{\circ} \mathrm{K}$.

\section{SUMMARY}

Some conclusions we draw from the $\mathrm{CO}$ observations are: (1) Molecular gas ( $\mathrm{CO}$ ) is present throughout the nuclear region although its abundance relative to $\mathrm{H}$ I falls sharply beyond 300 pc. (2) The kinematics and distribution of this gas might be described as nuclear disk plus expanding arms or a single spiral pattern reaching to within $100 \mathrm{pc}$ of the center, similar to that observed in Sc galaxies (3) The total mass of molecular gas within $600 \mathrm{pc}$ is probably $10^{7}-10^{8} M_{\odot}$, far exceeding the mass of $\mathrm{H} \mathrm{I}$. (4) Comparison of the $\mathrm{CO}$ and $100-\mu$ emission strongly suggests that the dust grains emitting in the far-infrared are located in the molecular regions.

This research was supported in part by the University of Minnesota Graduate School and by NSF grant GP-27983.

\section{REFERENCES}

Davis, J. H., and Vanden Bout, P. 1973, preprint.

Hoffmann, W. F., Frederick, C. L., and Emery, R. J. 1971, A p.J. (Letters), 164, L23.

Kerr, F. J., and Vallak, R. 1967, A ustralian J. Phys., A p. Suppl., No. 3.

McGee, R. X. 1970, Australian J. Phys., 23, 541.

Oort, J. 1970, Vatican Symposium on the Nuclei of Galaxies (Rome: Pontifica Academy), p. 11.

Rougoor, G. W. 1964, B.A.N., 17, 381.

Rougoor, G. W., and Oort, J. H. 1960, Proc. Nat. Acad.Sci., 46, 1.
Sanders, R. H., and Wrixon, G. T. 1973, Astr. and Ap., 26, 365.

Scoville, N. Z. 1972, A p. J. (Letters), 175, L127.

Scoville, N. Z., and Solomon, P. M. 1973, A p. J., 180, 31.

Scoville, N. Z., Solomon, P. M., and Penzias, A. A. 1974, in preparation.

Scoville, N. Z., Solomon, P. M., and Thaddeus, P. 1972, Ap. J., 172, 335.

Solomon, P. M., Scoville, N. Z., Jefferts, K. B., Penzias, A. A., and Wilson, R. W. 1972, A p.J., 178, 125. 